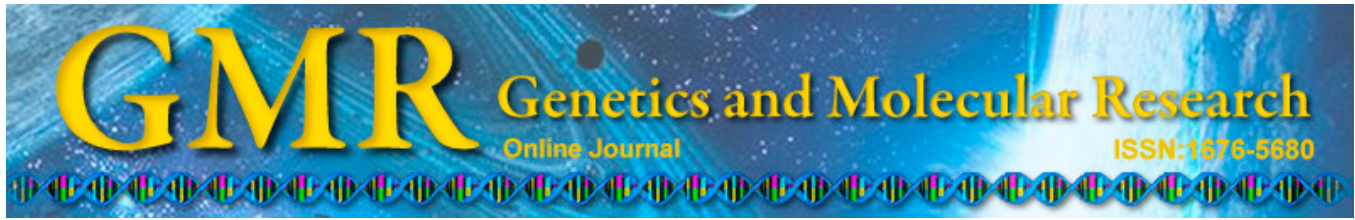

\title{
Comparison of DNA extraction methods for polymerase chain reaction amplification of guanaco (Lama guanicoe) fecal DNA samples
}

\author{
M.I. Espinosa ${ }^{1,2}$, A. Bertin ${ }^{1}$, F.A. Squeo ${ }^{1,3,4}$, A. Cortés ${ }^{1,3}$ and N. Gouin ${ }^{1,3,5}$ \\ ${ }^{1}$ Departamento de Biología, Facultad de Ciencias, Universidad de La Serena, \\ La Serena, Chile \\ ${ }^{2}$ Programa de Doctorado en Biología y Ecología Aplicada, \\ Universidad de La Serena, La Serena, Chile \\ ${ }^{3}$ Centro de Estudios Avanzados en Zonas Áridas, La Serena, Chile \\ ${ }^{4}$ Instituto de Ecología y Biodiversidad, Chile \\ ${ }^{5}$ Facultad de Ciencias del Mar, Universidad Cátolica del Norte, Coquimbo, Chile \\ Corresponding author: M.I. Espinosa \\ E-mail: maraines@gmail.com
}

Genet. Mol. Res. 14 (1): 400-406 (2015)

Received January 9, 2014

Accepted May 29, 2014

Published January 23, 2015

DOI http://dx.doi.org/10.4238/2015.January.23.13

\begin{abstract}
Feces-based population genetic studies have become increasingly popular. However, polymerase chain reaction (PCR) amplification rates from fecal material vary depending on the species, populations, loci, and extraction protocols. Here, we assessed the PCR amplification success of three microsatellite markers and a segment of the mitochondrial control region of DNA extracted from field-collected feces of guanaco (Lama guanicoe) using two protocols - Qiagen DNA Stool Kit and 2 cetyltrimethylammonium bromide/ phenol:chloroform:isoamyl alcohol (2CTAB/PCI) method. Chelex resin treatment to remove inhibitors was also tested. Our results show that the mitochondrial locus was the most difficult to amplify. PCR success rates improved for all markers after Chelex treatment of extracted DNA, and 2CTAB/PCI method (95.83\%) appeared to perform slightly better than
\end{abstract}


stool kit $(91.67 \%)$ for the nuclear markers. Amplification success was significantly influenced by the extraction method, Chelex treatment, and locus $(\mathrm{P}<0.001)$ but not by the freshness of the feces (fresh vs old, $\mathrm{P}=0.17$ ). The repeatability levels were high without Chelex treatment $(>0.89$ ), but they decreased slightly after treatment for amplification of nuclear markers and markedly after treatment for amplification of the mitochondrial control region. Thus, we showed that Chelex treatment gives high PCR success, especially for nuclear markers, and adequate DNA extraction rates can be achieved from $L$. guanicoe feces even from non-fresh fecal material. Although not significant, 2CTAB/PCI method tended to provide higher successful amplification rates on a whole set of samples, suggesting that the method could be particularly useful when using small sample sizes.

Key words: Non-invasive genetics; Mitochondrial control region; Microsatellites; Polymerase chain reaction inhibitors;

Chelex treatment; Lama guanicoe

\section{INTRODUCTION}

Non-invasive population genetics using field feces is a useful and advantageous method for investigating the ecology of elusive, rare, and endangered animal species. Indeed, the collection of non-invasive samples does not require the capture of specimens and is rather inexpensive (Fernando et al., 2003). Genetic analyses using fecal material and other tissues have been found to yield comparable results (Boston et al., 2012). Consequently, feces-based population genetic studies have become increasingly more popular. DNA extraction from feces, however, is usually more laborious than from other biological material because of the presence of large amounts of polymerase chain reaction (PCR) inhibitors in fecal material (Waits and Paetkau, 2005). In addition, the quality and quantity of fecal DNA is negatively affected by the exposure to local ambient conditions, the presence of digested food items, and natural degradation processes (Hájková et al., 2006). Herbivore feces, in particular, are known to contain larger quantities of PCR inhibitors than carnivore and omnivore feces (Banks et al., 2002), presumably because of the abundance of secondary metabolites derived from plants in herbivore feces; these metabolites can inhibit the PCR process and impede DNA amplification (Weising et al., 2005).

The DNA amplification success that is achieved from feces samples is variable regardless of whether the extraction is accomplished using commercial DNA extraction kits that are specifically designed for samples enriched with PCR inhibitors or DNA extraction protocols that are adapted from standard methods (Vallet et al., 2008). Species, geographical localization, diet, season, and PCR primers have all been found to influence the success of DNA amplification from fecal material (Hájková et al., 2006). The optimization of specific extraction protocols is thus critical to increase the number of successful amplifications from fecal material and to reach admissible amplification rates (Valière et al., 2007). In addition, optimized protocols usually allow reduced laboratory costs (Broquet et al., 2007).

The guanaco Lama guanicoe (Artiodactyla, Camelidae), a large South American herbivore, has faced dramatic population fragmentation during the last century because of 
anthropogenic habitat disturbance (Puig and Videla, 2007). This has resulted in severe risks of local and even regional extinction, especially throughout its northern distribution (Marín et al., 2008). Recent molecular studies support the hypothesis that northern guanaco populations harbor higher genetic diversity than those in the southern distribution (Maté et al., 2005; Marín et al., 2008). Nevertheless, knowledge about the evolutionary and ecological factors shaping the distribution and structure of the northern populations remains scarce. L. guanicoe presents various ecological and behavioral characteristics such as a wide home range, large escape distances, and low abundances that make observations and invasive sampling for genetic analysis very difficult in wild populations. Traditional methods such as field censuses and telemetry have been used in this species to estimate population sizes and home ranges (CONAF, 2009), but they often generate inaccurate results because of the limited number of individuals that can be counted or captured and the large extent of areas to be surveyed. The use of non-invasive sampling for population genetic studies can overcome all of these challenges by allowing the identification of individual organisms over extensive areas without having to handle animals (Waits and Paetkau, 2005). However, high amplification success rates of specific and hypervariable loci are necessary to achieve a fine resolution at the individual level using feces-like DNA sources, requiring a robust DNA extraction protocol. Marín et al. (2008) recommended the use of feces as an alternative source of DNA to investigate the phylogeography of the guanaco using commercially available extraction kits. However, to our knowledge, the effectiveness of such non-invasive sources of DNA from this herbivore species has not been reported. In this study, we assessed the PCR amplification success rate of three microsatellite markers and a segment of the mitochondrial DNA control region using DNA that was extracted from field-collected feces of $L$. guanicoe. We compared two well-established DNA extraction protocols: the Qiagen DNA Stool Kit and the 2 cetyltrimethylammonium bromide/phenol:chloroform:isoamyl alcohol (2CTAB/PCI) method, which are both reported to be capable of high levels of amplification success from feces in wild species (Vallet et al., 2008). We also tested the effect of a treatment with Chelex resin to remove inhibitors from extracted DNA using the concentrated Chelex resin treatment (CCT) that was proposed by Hebert et al. (2011).

\section{MATERIAL AND METHODS}

\section{Sampling}

Twenty-four fecal samples were collected from defecation sites of free-ranging guanacos in four sectors located in the north of Chile: Llanos de Challe National Park [N = 9; S28 13.775-W71 01.464; 0-100 $\mathrm{m}$ above sea level (masl)], Choros basin ( $=4$; S 2919.663-W 7113.810; 0-100 masl), Calvario Basin ( $\mathrm{N}=5$; S29 25.734-W70 29.189; 2700 masl), and Tres Quebradas Basin ( $=6$; S29 20.128-W70 06.936; 3400 masl) during summer 2012. Samples were categorized according to their degree of freshness. All samples that were collected immediately after observing the animal defecate or that still had anal jelly were categorized as fresh. In contrast, samples that were collected without observing the animal defecate, were hard to the touch, and were without anal jelly were categorized as old. Samples of both fresh and old feces were stored at ambient temperature in sterile tubes containing a $96 \%$ ethanol solution. 


\section{DNA extraction}

Two protocols were compared in our study: the QIAamp DNA Stool Mini Kit (QIAGEN, Hilden, Germany) following manufacturer recommendations and the 2CTAB/PCI extraction method that was proposed by Vallet et al. (2008), adapting the concentration of RNase to $50 \mathrm{mg} / \mathrm{mL}$ and resuspending the DNA pellet in water. With each extraction protocol, DNA extractions were performed in duplicate for each of the 24 feces samples. After DNA extraction, $20 \mu \mathrm{L}$ DNA solution was taken from each tube and treated using Chelex resin following the CCT method of Hebert et al. (2011).

\section{PCR amplification}

The amplification success was evaluated for each sample and method/treatment with three microsatellite and one mitochondrial loci. We used the three primer pairs that were developed by Lang et al. (1996) to amplify the microsatellites YWLL08 (135-177 bp), YWLL29 (218-232 bp), and YWLL46 (98-110 bp). A 600-bp segment of the mitochondrial DNA (mtDNA) control region was amplified using primers Lloop0007G (5'-GTACTAAAAGAA AATATCATGTC-3') and Hloop550G (5'-ATGGACTGAATAGCACCTTATG-3'), which are specific to artiodactyls (Marín et al., 2008). PCR amplifications of the microsatellite loci were performed in a total volume of $25 \mu \mathrm{L}$ containing $50 \mathrm{ng}$ DNA, $0.8 \mathrm{mM}$ dNTPs, 0.2 $\mathrm{mM}$ primers, $2 \mathrm{mM} \mathrm{MgCl}$, $1 \mathrm{U}$ Platinum Taq DNA Polymerase (Invitrogen), and 1X PCR Buffer (Invitrogen). The same conditions were used for the mtDNA control region, except that $0.3 \mathrm{mM}$ primers and $2.5 \mathrm{mM} \mathrm{MgCl}{ }_{2}$ were used. The amplification cycling program for the microsatellite loci was as follows: denaturation for $2 \mathrm{~min}$ at $95^{\circ} \mathrm{C} ; 40$ cycles of $45 \mathrm{~s}$ at $94^{\circ} \mathrm{C}, 45$ $\mathrm{s}$ at $54^{\circ} \mathrm{C}$, and $45 \mathrm{~s}$ at $72^{\circ} \mathrm{C}$; and a final extension of $15 \mathrm{~min}$ at $72^{\circ} \mathrm{C}$. For the control region, we used an initial denaturation step of $5 \mathrm{~min}$ at $95^{\circ} \mathrm{C} ; 35$ cycles of $45 \mathrm{~s}$ at $95^{\circ} \mathrm{C}, 30 \mathrm{~s}$ at $50^{\circ} \mathrm{C}$, and $45 \mathrm{~s}$ at $72^{\circ} \mathrm{C}$; and a final extension of $5 \mathrm{~min}$ at $72^{\circ} \mathrm{C}$. Each PCR amplification was carried out twice for each tested marker in order to evaluate the amplification robustness. PCR products were visualized on 1.8\% agarose gels stained in GelRed (Biotium).

To account for potential contamination, positive controls using DNA extracted from skin tissue from guanaco remains that were found in the field and negative controls with only extraction reagents and sterile water were performed in each run of both extraction and amplification reactions.

\section{Statistical analyses}

Repeatability indices were calculated for each marker using a generalized linear mixed model (GLMM) with additive overdispersion implemented in the R package "rptR". The potential effect of the extraction method, CCT, feces state (old/fresh), and locus on PCR success rates was evaluated through a GLMM considering individuals as a random effect. This analysis was performed using the R package "Ime4". The significance of the fixed effects was assessed by conducting $\chi^{2}$ likelihood ratio tests.

\section{RESULTS AND DISCUSSION}

A total of 768 amplification reactions were conducted. Amplification success rates 
varied significantly between markers (loci effect: $\chi^{2}=62.87$; d.f. $=3 ; \mathrm{P}<0.001$ ). Globally, the $\sim 600$-bp portion of the mtDNA control region was more difficult to amplify than the microsatellite markers (Table 1 and Figure 1), possibly because its size was larger than the others. The locus size has been shown to influence amplification success in population genetic studies using non-invasive samples, with larger PCR products usually displaying lower amplification rates because of DNA template degradation by environmental factors and microorganisms (Broquet et al., 2007). The CCT led to a marked improvement in amplification success rates (CCT effect: $\chi^{2}=57.03$; d.f. $=1 ; \mathrm{P}<0.001$ ) both for the nuclear and mtDNA loci (Figure 1 and Table 1).

\section{Table 1. Repeatability index (means \pm SD) and average PCR success rates for each method and treatment.}

\begin{tabular}{|c|c|c|c|c|}
\hline \multirow[t]{2}{*}{ Locus } & \multicolumn{2}{|c|}{ 2CTAB/PCI } & \multicolumn{2}{|c|}{ Stool Kit } \\
\hline & Without CCT & With CCT & Without CCT & With CCT \\
\hline YWLL08 & $0.98 \pm 0.06$ & $0.98 \pm 0.02$ & $0.93 \pm 0.13$ & $0.90 \pm 0.07$ \\
\hline YWLL29 & $0.89 \pm 0.09$ & $0.94 \pm 0.21$ & $0.96 \pm 0.07$ & $0.95 \pm 0.12$ \\
\hline YWLL46 & $0.93 \pm 0.12$ & $0.80 \pm 0.28$ & $0.98 \pm 0.05$ & $0.95 \pm 0.26$ \\
\hline Average repeatability & 0.93 & 0.91 & 0.96 & 0.93 \\
\hline Average PCR success (\%) & 68.06 & 95.83 & 80.56 & 91.67 \\
\hline Control region & $0.89 \pm 0.22$ & $0.61 \pm 0.24$ & $0.97 \pm 0.16$ & $0.81 \pm 0.25$ \\
\hline Average PCR success (\%) & 29.17 & 62.50 & 77.08 & 81.25 \\
\hline
\end{tabular}

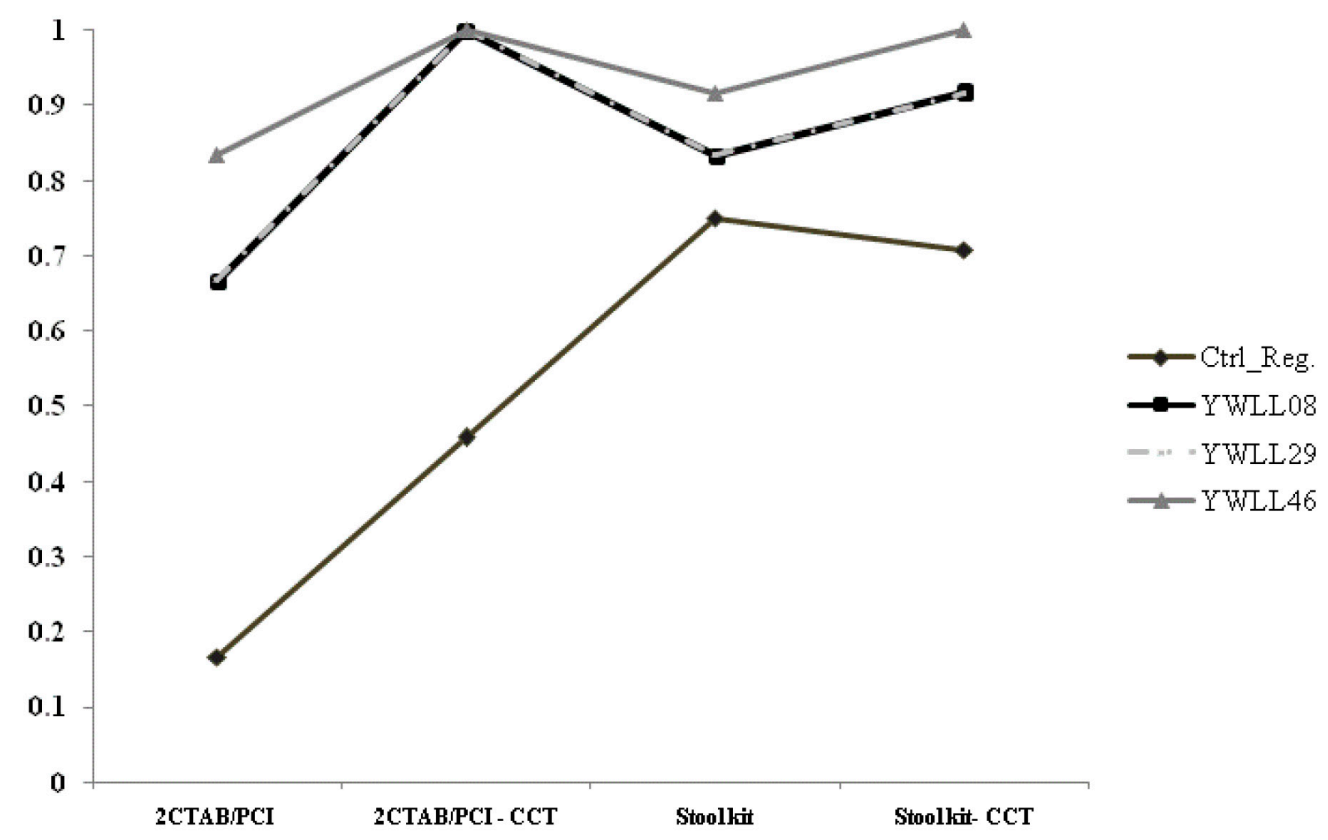

Figure 1. Rates of amplified samples after two independent PCRs for each locus and DNA extraction method (2CTAB/PCI and Qiagen Stool Kit) with and without Chelex treatment (CCT). 
Indeed, the amplification success of the 2CTAB/PCI and Qiagen Stool Kit was increased on average by about 28 and $11 \%$, respectively, for the nuclear markers, and by about 33 and $4 \%$, respectively, for the mitochondrial control region (Table 1). PCR success rates with CCT also varied between marker types (loci effect: $\chi^{2}=41.25$; d.f. $=3 ; \mathrm{P}<0.001$ ). For the nuclear markers, the $2 \mathrm{CTAB} / \mathrm{PCI}$ method harbored slightly higher amplification rates than the stool kit $(+4.2 \%)$. This method, however, was less successful with the mtDNA locus and resulted in lower amplification rates than the stool kit (-19\%; Table 1). Despite leading to higher amplification success, CCT reduced PCR repeatability; the repeatability was reduced slightly for the nuclear markers $(2-3 \%)$, but the reduction was much more pronounced for the mtDNA locus (29\% for the 2CTAB/PCI method and $16 \%$ for the stool kit; Table 1). Overall, the average repeatability remained high and nearly identical for the nuclear markers with both DNA extraction methods ( $\geq 0.91$; Table 1$)$. It is possible that DNA is further degraded during CCT, which makes the amplification of the $~ 600-b p$ mitochondrial DNA fragment even more difficult. The use of new primers to amplify smaller portions of this locus might be a good alternative under these conditions. Therefore, our study shows that the Chelex treatment that was proposed by Hebert et al. (2011) improves PCR amplification success of nuclear markers such as microsatellites from field-collected guanaco feces samples. Although the PCR success rate of the nuclear markers after CCT was only slightly higher with the 2CTAB/PCI method than with the stool kit, only the former protocol allowed the amplification of all three microsatellite markers from all samples and duplicate amplifications (Figure 1). With the stool kit and CCT combination, such a success level was reached for the YWLL46 microsatellite only. This suggests that the 2CTAB/PCI-CCT protocol could be more successful than the stool kitCCT protocol to provide suitable DNA for PCR amplification of an entire set of microsatellite markers. Therefore, this protocol could be particularly useful when sample sizes are small.

While our analyses revealed that the extraction method, CCT, and locus had significant effects on PCR success rates ( $P<0.001$ in all cases), the freshness of the feces did not (feces state effect: $\chi^{2}=0.75$; d.f. $=1 ; \mathrm{P}=0.38$ ), suggesting that old feces were as efficient as fresh feces in generating PCR products. While feces freshness is usually considered to be an essential determinant of amplification success (Brinkman et al., 2011), other studies in ungulates and otters have failed to relate the DNA amplification success from fecal material with feces age (Hájková et al., 2006; Lampa et al., 2008; Brinkman et al., 2010). In particular, climatic conditions and air moisture may play an important role in DNA preservation. For example, Brinkman et al. (2010) reported high degradation of fecal DNA that was exposed to rainfall while Piggott (2005) obtained higher DNA quality from feces samples that were collected during summer. Because of the dry climatic conditions of our study region (Di Castri and Hajek, 1976), DNA degradation in L. guanicoe feces might be relatively slow and facilitate the amplification of small PCR products from non-fresh fecal material.

In conclusion, we found that the removal of inhibitors from DNA that was extracted from guanaco feces using a Chelex treatment led to higher PCR success, especially for nuclear markers, and that DNA can be successfully amplified from feces samples from northern Chile even when the samples are not fresh. This expands the window of opportunities for molecular studies in L. guanicoe in this region where populations are estimated to be small and strongly affected by anthropogenic threats. In this context, the 2CTAB/PCI method with CCT might be particularly adequate because this protocol appeared to hold the greatest promise in providing PCR products from a whole set of samples. 


\section{ACKNOWLEDGMENTS}

We thank the staff of the Llanos de Challe National Park (CONAF - Atacama), the Community of Los Choros, and the Environmental Department of Nevada Mining Company for their assistance in the field. Research supported by a grant from the South America-Barrick Gold Corporation and the Institute of Ecology and Biodiversity (IEB).

\section{REFERENCES}

Banks SC, Piggott MP, Hansen BD, Robinson NA, et al. (2002). Wombat coprogenetics: enumerating a common wombat population by microsatellite analysis of faecal DNA. Aust. J. Zool. 50: 193-204.

Boston ES, Puechmaille SJ, Scott DD, Buckley DJ, et al. (2012). Empirical assessment of non-invasive population genetics in bats: comparison of DNA quality from faecal and tissue samples. Acta Chiropterol. 14: 45-52.

Brinkman TJ, Schwartz MK, Person DK, Pilgrim KL, et al. (2010). Effects of time and rainfall on PCR success using DNA extracted from deer fecal pellets. Conserv. Genet. 11: 1547-1552.

Brinkman TJ, Person DK, Chapin FS, Smith W, et al. (2011). Estimating abundance of Sitka black-tailed deer using DNA from fecal pellets. J. Wildlife Manage. 75: 232-242.

Broquet T, Ménard N and Petit E (2007). Noninvasive population genetics: a review of sample source, diet, fragment length and microsatellite motif effects on amplification success and genotyping error rates. Conserv. Genet. 8: 249-260.

CONAF (Corporación Nacional Forestal) (2009). Informe censo estival del guanaco (Lama guanicoe). Parque Nacional Llanos de Challe, Región de Atacama. CONAF, Chile.

Di Castri F and Hajek ER (1976). Bioclimatología de Chile. Vicerrectoría Académica de la Universidad Católica de Chile, Santiago.

Fernando P, Vidya TNC, Rajapakse C, Dangolla A, et al. (2003). Reliable noninvasive genotyping: fantasy or reality? $J$. Hered. 94: 115-123.

Hájková P, Zemanová B, Bryja J, Hájek B, et al. (2006). Factors affecting success of PCR amplification of microsatellite loci from otter faeces. Mol. Ecol. Notes 6: 559-562.

Hebert L, Darden SK, Pedersen BV and Dabelsteen T (2011). Increased DNA amplification success of non-invasive genetic samples by successful removal of inhibitors from faecal samples collected in the field. Conserv. Genet. Resour. 3: 41-43.

Lampa S, Gruber B, Henle K and Hoehn M (2008). An optimisation approach to increase DNA amplification success of otter faeces. Conserv. Genet. 9: 201-210.

Lang KDM, Wang Y and Plante Y (1996). Fifteen polymorphic dinucleotide microsatellites in llamas and alpacas. Anim. Genet. 27: 293.

Marín JC, Spotorno AE, González BA, Bonacic C, et al. (2008). Mitochondrial DNA variation and systematics of the guanaco (Lama guanicoe, Artiodactyla: Camelidae). J. Mammal. 89: 269-281.

Maté ML, Bustamante A, Giovambattista G, De Lamo D, et al. (2005). Genetic diversity and differentiation of guanaco populations from Argentina inferred from microsatellite data. Anim. Genet. 36: 316-321.

Piggott MP (2005). Effect of sample age and season of collection on the reliability of microsatellite genotyping of faecal DNA. Wildlife Res. 31: 485-493.

Puig S and Videla F (2007). Distribución, densidades y estado de conservación de los camélidos. Diversidad Biológica y Cultural de los Altos Andes Centrales de Argentina, 198.

Valière N, Bonenfant C, Toïgo C, Luikart G, et al. (2007). Importance of a pilot study for non-invasive genetic sampling: genotyping errors and population size estimation in red deer. Conserv. Genet. 8: 69-78.

Vallet D, Petit EJ, Gatti S, Levréro F, et al. (2008). A new 2CTAB/PCI method improves DNA amplification success from faeces of Mediterranean (Barbary macaques) and tropical (lowland gorillas) primates. Conserv. Genet. 9: 677-680.

Waits LP and Paetkau D (2005). Noninvasive genetic sampling tools for wildlife biologists: a review of applications and recommendations for accurate data collection. J. Wildlife Manage. 69: 1419-1433.

Weising K, Nybom H, Wolff K and Kahl G (2005). DNA fingerprinting in plants: principles, methods, and applications. CRC Press, Boca Raton, 88-94. 